\title{
Der familiäre Hintergrund „suchtbelastete Familie“ und dessen Auswirkung auf Mentalisierungsprozesse fremduntergebrachter Kinder
}

\author{
Nadja Springer · Brigitte Lueger-Schuster
}

Online publiziert: 13. Mai 2020

(C) Der/die Autor(en) 2020

\begin{abstract}
Zusammenfassung Studien über Bindung weisen auf Schwierigkeiten drogenabhängiger Eltern hinsichtlich ihrer Reaktion auf die emotionalen Signale der Kinder und die daraus abzuleitenden Verhaltensweisen hin. Dies lässt auf eine reduzierte Mentalisierungsfähigkeit schließen. Mentalisieren bedeutet die grundlegende menschliche Fähigkeit, Verhalten in Bezug auf Gedanken und Gefühle zu verstehen.

Um diese Fähigkeit bei fremduntergebrachten Kinder (6-12 Jahre) aus suchtbelasteten Familien und deren aktuellen Bezugspersonen zu untersuchen, wurde eine auf dem Konzept der Mentalisierung basierende Gruppenintervention entwickelt, die das Wissen über Drogenabhängigkeit erweitern und soziale und affektive Fähigkeiten verbessern soll.

Erhebungsinstrumente: Skala des Reflective Functioning (RF-Score) für das Adult Attachment Interview und für das Child Attachment Interview, Inventar der Sozialkompetenzen (ISK), Coloured Progressive Matrices (CPM), Child Behavior Checklist (CBCL/618R), „Patte-Noire“-Test, Einstellungen zum Drogenkonsum (EBDD Bewertungsinstrument).

An Hand zweier Fallbeispiele aus dieser laufenden Pilotstudie, werden der diagnostische Prozess sowie beobachtbare und messbare Veränderungen über den Untersuchungszeitraum von mehr als 12 Monaten exemplarisch dargestellt und diskutiert.
\end{abstract}

\footnotetext{
N. Springer $(\bowtie)$

Verein Dialog, Wien, Österreich

nadja.springer@dialog-on.at

B. Lueger-Schuster

Institut für Klinische- und Gesundheitspsychologie, Fakultät für Psychologie, Universität Wien, Wien, Österreich
}

Schlüsselwörter Mentalisierung · Reflexive Funktion - Suchtbelastete Familie · Gruppenintervention · Fremdunterbringung • Fallstudie

The impact of parental licit/illicit drug abuse background on mentalization processes in children living in foster care

Summary Studies on attachment show difficulties for parents with drug-use disorders in responding to the emotional signals of their children. These difficulties suggest a reduced capacity for mentalization. Mentalization is the basic human ability to understand behavior in terms of mental states such as thoughts and feelings.

In order to investigate this ability of externally placed children (6-12 years) from drug abusing families and their current caregivers, a group intervention based on the concept of mentalization has been developed, which aims to expand knowledge about drug addiction and improve social and affective skills.

The survey instruments used are: reflective Functioning Scale (RF-Score) for the Adult Attachment Interview and for the Child Attachment Interview, Social Competence Inventory (ISK), Colored Progressive Matrices (CPM), Child Behavior Checklist (CBCL/6-18R), "Patte-Noire" test, attitudes towards drug use (EMCDDA assessment tool).

Based on two case studies from this ongoing pilot study, the diagnostic process and observable and measurable changes over the study period of more than 12 months are presented and discussed.

Keywords Mentalization · Reflective functioning · Parental drug abuse · Group intervention · Foster care $\cdot$ Case studies 


\section{Konzept der Mentalisierung}

Überlegungen zur Mentalisierung wurden 1989 eingeführt und später im Rahmen einer großen empirischen Studie zu Bindungsstilen im Kindesalter als Konzept entwickelt (Fonagy et al. 1991).

Die Fähigkeit zur Mentalisierung spielt eine entscheidende Rolle als Schutzfaktor gegen Traumatisierungen. Durch die kognitive und emotionale Kapazität der internen Regulierung von Emotionen sind Personen erfolgreicher, Gefühle von Ärger, Angst, Scham und Traurigkeit, die aus Traumata resultieren, zu tolerieren (Allen et al. 2008; Ensink et al. 2017).

Bolm (2010, S. 59) betont, dass sich eine „besonders gute Mentalisierungsfähigkeit im Vermögen zum phantasievollen, klugen und vorausschauenden Umgang mit dem Wechselspiel von Innen- und Außenwelt zeigt“. Fonagy (1995) nennt diese Fähigkeit "playing with reality“ als Referenz zu Winnicott (1971), der das Spielen als eine Entwicklungsleistung betrachtete. Es bereichert nicht nur das Leben, sondern unterstützt auch die phantasievolle Ausarbeitung von Gedanken und Gefühlen über Körper, Objektbeziehungen und Ängste. Die Mentalisierungsfähigkeit von Kindern lässt sich am besten während der Latenzzeit beobachten. Eine der wichtigsten Herausforderungen der Latenz besteht darin, dass die Intensität der Bindung an die Eltern aufgelöst und verdrängt wird. Erwachsene und Kinder außerhalb der Familie nehmen eine zunehmend größere Rolle im Leben des Kindes ein. Die Bewältigung dieser Herausforderungen hängt vom Grad der Entwicklung der Ich-Funktionen ab, wie z.B. eine effiziente emotionale Kontrolle und die Fähigkeit der Übernahme der Perspektive des Anderen (Dejko et al. 2016). Mentalisationsbasierte Interventionen zur Verbesserung der Ich-Funktionen werden bereits bei verschiedenen Indikationen wie z.B. bei Persönlichkeitsstörungen und Drogenabhängigkeit eingesetzt (z. B. Bateman und Fonagy 2008; Suchman et al. 2010).

Die vorliegende Pilotstudie konzentriert sich auf die Operationalisierung des Mentalisierungsmodells mittels der Reflective Functioning Scale (RFS). Der Begriff "reflexive Funktion“ (RF) bezieht sich auf die Operationalisierung psychischer Prozesse, die der Mentalisierungsfähigkeit zugrunde liegen (Fonagy et al. 1998).

Die Fähigkeit der primären Bezugsperson, über den Verstand ihres Kindes nachzudenken, wird entweder als elterlicher Verstand, Einsicht oder reflexive Funktion (RF), bezeichnet. Diese kann bei Erwachsenen und bei Kindern mit Hilfe des Erwachsenen/KindBindungsinterviews (AAI/CAI) beurteilt werden.

Zwar gibt es Forschungsarbeiten zur Mentalisierung bei drogenmissbrauchenden Müttern, doch wurde darauf hingewiesen, dass die Forschung zur Mentalisierungsfähigkeit bei Kindern von drogenmissbrauchenden Eltern noch immer fehlt und, dass es an Interventionen für fremduntergebrachte Kin- der und deren Bezugspersonen mangelt, die sich mit ihren Erfahrungen und Gefühlen im Zusammenhang mit der Fremdunterbringung befassen (Ostler et al. 2010; Suchman et al. 2010). Eine Metaanalyse über bindungsorientierte Interventionen von BekermansKranenburg et al. (2003) zeigte, dass effektive Interventionen eine moderate Anzahl von Sitzungen (5-16) aufweisen und in ihren Zielen sehr fokussiert sind. Sowohl in der Gruppenintervention für die Kinder, wie auch in der Arbeit mit den Betreuer*innen gilt es $\mathrm{zu}$ vermitteln welch grundlegende Bedeutung es für ein gegenseitiges Verstehen hat, zu mentalisieren und darum, diese Fähigkeit zu verbessern. Zu den verwendeten Techniken zählt einerseits im Gruppensetting Mentalisieren zu üben, andererseits aber auch die Aufmerksamkeit auf Situationen zu richten, an denen die Mentalisierung möglicherweise zusammenbricht (Midgley et al. 2017).

\section{„Mentalisieren" als therapeutische Grundhaltung}

$\mathrm{Zu}$ den Schlüsselkomponenten der mentalisierenden Haltung der Psychotherapeut*innen gehören Neugierde, Humor und das Interesse an der Erforschung der Perspektive des/der Anderen durch eine konstante Berücksichtigung des kognitiven und affektiven Zustands der Teilnehmer*innen.

\section{Fremduntergebrachte Kinder: Psychosoziale und psychodynamische Ansätze}

Die eigene Familiengeschichte der Eltern und deren Überzeugungen über Emotionen und emotionalen Ausdruck beeinflussen die Art und Weise, wie Eltern auf emotionaler Ebene mit ihren Kindern interagieren (Thomsen et al. 2017). Ein vergleichbarer Mechanismus steuert die Beziehung zwischen Betreuer*innen und ihren Schützlingen.

Misshandlung, Vernachlässigung und der Wechsel in die Fremdunterbringung sind traumatische Ereignisse, die die unmittelbare und zukünftige psychosoziale Entwicklung und psychische Gesundheit von Kindern beeinflussen können (Bowlby 1998; Cicchetti et al. 2006). Wie gut Kinder diese körperlichen, emotionalen oder mentalen Traumata überstehen, hängt stark von der Qualität der aktuellen Beziehungen ab. Unbewältigte Traumata haben einen großen Einfluss auf das Bindungsverhalten von fremduntergebrachten Kindern gegenüber ihren Bezugspersonen (Muller et al. 2012).

Latenzkinder sind in ihrer Entwicklung von den reflexiven Funktionsfähigkeiten ihrer Eltern abhängig (Jemerin 2004). In der Regel sind die Eltern in der Lage, die Gefühle des Kindes zu ertragen, können dem Gefühl durch Spiegelung eine Bedeutung geben und gegebenenfalls die Situation oder das Gefühl neu einordnen. Durch die Wiederholung nonverbaler Erfahrungen innerhalb der Eltern-Kind-Beziehung kann das Kind von dieser Art der Interaktion profitie- 
ren, indem es lernt, sich verstanden und anerkannt $\mathrm{zu}$ fühlen. Die Reflexive Funktion ist eine Entwicklungsleistung, die es Kindern ermöglicht, nicht nur situationsbezogen auf das Verhalten anderer $\mathrm{zu}$ reagieren, sondern über das sichtbare Verhalten hinaus dem anderen Gefühle, Absichten und Wünsche zuzuschreiben. Das Kind lernt die mentalen Zustände anderer $\mathrm{zu}$ „lesen“ und macht dadurch Handlungen für sich bedeutungsvoll und vorhersehbar.

Betreuer*innen und Pflegeeltern haben oft Schwierigkeiten, das Verhalten der Kinder zu entschlüsseln, weil die nicht gemeinsam verbrachte Zeit vor der Fremdunterbringung oder der Mangel an Informationen über die Lebensumstände der leiblichen Eltern dies verhindern. Die Betreuungsperson und das Kind selbst sind über die Geschichte des/der Anderen oft unzureichend informiert. Daraus resultieren Gefühle der Hilflosigkeit auf Seiten der Betreuer*innen, weil sie nicht in der Lage sind, die Probleme der Kinder zu lindern.

Selbst wenn das Kind wenig bis keine Hintergrundinformationen über seine Ursprungsfamilie hat, wird es implizit eine Biographie erstellen, die weitgehend auf seinen Phantasien beruht. In diesem „Familienroman“ (Freud 1909) werden die biologischen Eltern entweder idealisiert oder entwertet. Dies kann sich auf verschiedene Weise manifestieren, oftmals durch ein Verhalten, das darauf abzielt, ein weiteres Verlassen werden zu vermeiden (Muller et al. 2012).

\section{Studiendesign}

Die Datenerhebung für diese explorative Pilotstudie im Mixed Methods Design findet im Rahmen eines Praxisprojekts im städtischen Umfeld statt: Der Verein Dialog bietet in Kooperation mit dem Institut für Erziehungshilfe eine speziell für diese Zielgruppe entwickelte, mentalisierungsbasierte Gruppenintervention für fremduntergebrachte Kinder aus suchtbelasteten Familien an. Durch die Kooperation zweier im Bereich der Suchthilfe und im Bereich der Erziehungshilfe anerkannter Institutionen, kommt es bei diesem Angebot zu einer optimalen Nutzung der vorhandenen Kompetenzen:

1. 10-Einheiten à 90 min für fremduntergebrachte Kinder zur Erweiterung des Wissens über Drogenabhängigkeit und zur Förderung sozialer und affektiver Kompetenzen

2. Informationsabende für Bezugsbetreuer*innen während des Interventionsprogramms beinhalten Informationen über den Hintergrund des elterlichen legalen/illegalen Drogenmissbrauchs mit Schwerpunkt auf die Betreuer*in-Kind-Interaktion.

$\mathrm{Zu}$ den Forschungsfragen der Pilotstudie gehören die Auswirkungen des Zeitpunkts und der Art der Fremdunterbringung auf die Entwicklung von Mentalisierungsprozessen, die psychische Gesundheit des
Kindes sowie die Einstellung der Betreuer*innen zum Drogengebrauch.

\section{Beschreibung der Stichprobe}

Fremduntergebrachte Kinder aus suchtbelasteten Familien sowie deren Haupt-Bezugspersonen, die an der Gruppenintervention teilnehmen, werden gebeten, an dieser laufenden Pilotstudie zu partizipieren.

Die Datenerhebung wird von klinischen Psycholog*innen und Psychotherapeut*innen durchgeführt. Sie erfolgt nach Unterzeichnung der Einverständniserklärung durch die Betreuer*in. Folgende Einschlusskriterien gelten für die Studie: (1) das Kind ist aufgrund von elterlichem legalen und/oder illegalen Drogenmissbrauch fremduntergebracht und (2) das Kind ist zwischen 6 und 12 Jahre alt.

\section{Verwendete Instrumente}

Folgende standardisierte, psychologische Testverfahren kommen zum Einsatz:

- Adult Attachment Interview (Adult Reflective Functioning Scale),

- Child Attachment Interview (Child Reflective Functioning Scale),

- Child Behavior Checklist CBCL 6-18,

- Raven's Matrizentest CPM,

- Schweinchen Schwarzfuß-Test („patte noire“)

- Sekundärskala „Reflexibilität“ des Inventar sozialer Kompetenzen (ISK)

und ein von der Europäischen Bobachtungsstelle für Drogen und Drogensucht (EBDD) entwickelter Fragebogen zu allgemeinen Einstellungen zum Drogenkonsum (European Monitoring Center for Drugs and Drug Addiction 2020).

Die Datenerhebung erfolgt zu drei Testzeitpunkten:

$\mathbf{T}_{\mathbf{0}}$ (vor der Gruppenintervention): Biografisches Interview; AAI; CAI; CBCL/6-18R; CPM; „patte noire“; ISK; Einstellung zum Drogenkonsum (Fragebogen der EBDD)

$\mathbf{T}_{\mathbf{1}}$ (innerhalb 1 Monats nach Beendigung der Intervention): CAI; CBCL/6-18R; „patte noire“; CPM

$\mathbf{T}_{\mathbf{2}}$ (12 Monate nach Beendigung der Intervention): CAI; CBCL/6-18R; „patte noire“; CPM

Relevante biographische Daten, wie: Alter bei der 1. Fremdunterbringung, Abhängigkeitssyndrom der biologischen Eltern, Anzahl der Betreuer*innen seit der Fremdunterbringung bzw. Anzahl der Pflegefamilien, werden durch geschlossene Fragen erhoben.

Auf ein Design mit einer Kontrollgruppe wird in dieser Pilotstudie verzichtet. Es kann nicht garantiert werden, dass Kinder, die zunächst auf eine Warteliste gesetzt werden (dabei als Kontrollgruppe dienen), tatsächlich eine Intervention erhalten werden. Eben- 


\begin{tabular}{|c|c|c|c|c|c|c|c|c|c|c|c|c|c|}
\hline \multicolumn{4}{|c|}{ Bezugsbetreuer_*in } & \multicolumn{10}{|c|}{ Kind } \\
\hline & $*$ & $* *$ & $* * *$ & & t0* & $\mathrm{t} 1^{*}$ & $\mathrm{t} 2^{*}$ & $\mathrm{t} 0 * * * *$ & $\mathrm{t} 1 * * * *$ & $\mathrm{t} 2 * * * *$ & $\mathrm{t} 0$ ***** & $\mathrm{t} 1$ ***** & $\mathrm{t} 2$ ****** \\
\hline Marie & 3 & 2 & 3 & Anna & 4 & 4 & 6 & $\begin{array}{l}\text { (E) } \mathrm{T}=60 \\
\text { (I) } \mathrm{T}=69\end{array}$ & $\begin{array}{l}\text { (E) } \mathrm{T}=60 \\
\text { (I) } \mathrm{T}=69\end{array}$ & $\begin{array}{l}\text { (E) } \mathrm{T}=59 \\
\text { (I) } \mathrm{T}=70\end{array}$ & II & III & II \\
\hline Katja & 3 & 2 & 2,17 & David & 2 & 5 & 4 & $\begin{array}{l}\text { (E) } \mathrm{T}=56 \\
\text { (I) } \mathrm{T}=61\end{array}$ & $\begin{array}{l}\text { (E) } \mathrm{T}=58 \\
\text { (I) } \mathrm{T}=61\end{array}$ & $\begin{array}{l}\text { (E) } \mathrm{T}=60 \\
\text { (I) } \mathrm{T}=62\end{array}$ & IV & IV & IV \\
\hline
\end{tabular}

Abb. 1 Deskriptive Darstellung der Ergebnisse (t0,t1,t2). $\mathrm{AAl} / \mathrm{CAl}^{*}-\mathrm{RF}-\mathrm{Score}:-1$ = kein RF; $\leq 3$ niedriger RF; $4-6$ durchschnittlicher RF; $\geq 7$ hoher RF; ISK ${ }^{\star \star}$ - Sekundärskala „Reflexibilität": unterdurchschnittlich =1; durchschnittlich = 2; überdurchschnittlich $=3$; Einstellungen zum Drogenkonsum ${ }^{\star * *}$ :

sowenig gibt es die Möglichkeit des Vergleichs mit einer TAU-Gruppe (treatment as usual), da die Kinder in den unterschiedlichen Settings lebend, nicht nur sehr unterschiedliche Erfahrungen mit psychologischen bzw. psychotherapeutischen Angeboten haben, sondern auch der Zugang zu diversen, unterstützenden Angeboten unterschiedlich ist.

\section{Fallbeispiele}

Die Auswahl der Fälle ist in der divergenten Entwicklung dieser beiden Kinder trotz ähnlicher, biographischer Ausgangssituation begründet. Beide Kinder wurden erst nach dem 2. Lebensjahr fremduntergebracht und haben seither den Lebensmittelpunkt in einer Wohngemeinschaft (WG). Die leiblichen Eltern sind polytoxikoman und in beiden Familien gibt es Hafterfahrungen von zumindest einem Elternteil. Alle Namen sind anonymisiert.

Bevor die beiden Fälle im Detail vorgestellt werden, gibt Abb. 1 einen Überblick über die Ergebnisse der verwendeten Instrumente zu den drei Testzeitpunkten. Im Verlauf der Falldarstellungen und der Diskussion wird auf die Ergebnisse Bezug genommen.

\section{Anna}

Anna ist $\mathrm{zu} \mathrm{T}_{0}$ neun Jahre alt und lebt seit ihrem dritten Lebensjahr gemeinsam mit ihrem älteren Bruder in einer WG. Beide biologischen Eltern sind polytoxikoman. Die Abnahme erfolgte aufgrund eines Haftantritts der leiblichen Mutter. Der Kontakt zu den getrenntlebenden Eltern hat sich in den ersten Jahren auf Telefonate beschränkt. Seit 2016 gibt es Besuchskontakte, seit 2018 darf Anna die Wochenenden regelmäßig alternierend bei ihrer Mutter und ihrem Vater verbringen. Anna hat die WG nicht gewechselt, die Bezugsbetreuung änderte sich innerhalb des Aufenthalts jedoch bereits siebenmal. 2016-2018 wurde sie von Marie betreut. Seit 2019 hat sie wieder eine neue Bezugsbetreuerin.

Marie ist $\mathrm{zu} \mathrm{T}_{0} 22$ Jahre alt. Bei dem Fragebogen der EBDD steht der erzielte Wert für eine weder sehr liberale noch restriktive Einstellung zum Drogenkon-
$1=$ negative Einstellung ; $5=$ positive Einstellung; $\mathrm{CBCL}^{\star * * *}$ internale/externale Problemskalen T-Werte: $<60$ unauffällig; 60-63 Grenzbereich; >63 Cut-off-Wert für klinische Auffälligkeit $\mathrm{CPM}^{\star \star \star \star \star *}$ : Leistungsstufen I-IV nach Prozentrang (PR): I: $P R \geq 95$; II: PR 75-94; III: PR 25-74; IV PR 6-24; V PR $\leq 5$

sum. Die Mentalisierungsfähigkeit (RF-Score im AAI) ist zwar vorhanden, es zeigen sich einige Hinweise auf die Berücksichtigung mentaler Zustände, die meisten Hinweise werden jedoch nicht explizit gemacht. Ein ähnliches Bild zeigt sich in der Sekundärskala „Reflexibilität“ des ISK. Der Wert im unteren Durchschnittsbereich bedeutet, dass sie sich mit ihrem Verhalten und dem Erleben des Verhaltens der Anderen in einem nicht besonders hohen Maß auseinandersetzt.

Anna ist $\mathrm{zu} \mathrm{T}_{0}$ neugierig jedoch unsicher. Ihre Unsicherheit zeigt sich im Ergebnis des CPM, wo sie im Vergleich zu den beiden anderen Testzeitpunkten schlechter abschneidet. Die internalen Probleme, die Anna zu allen drei Testzeitpunkten zeigt (CBCL), liegen alle im ängstlich/depressiven Bereich. Das Child Attachment Interview ist für Anna $\mathrm{zu} \mathrm{T}_{0}$ und $\mathrm{zu} \mathrm{T}_{1}$ eine deutliche, psychische Belastung. In der kognitiven und affektiven, reflexiven Auseinandersetzung mit ihrer Familie zeigt sie eine Idealisierung der biologischen Eltern und eine Entwertung ihrer eigenen Person. Sie berichtet von selbstverletzendem Verhalten in den vergangenen Jahren. $\mathrm{Zu} \mathrm{T}_{3}$ ist Anna deutlich ruhiger und klarer in der Differenzierung zwischen ihrem Erleben der wichtigen Bezugspersonen und ihren eigenen Bedürfnissen. Zu den beiden ersten Testzeitpunkten enthält das Interview mehrere Beispiele für spontane Berichte über ihren psychischen $\mathrm{Zu}$ stand. Die Reflexive Funktion ist jedoch noch nicht fest als primärer Modus ihres Verständnisses von Erfahrungen etabliert. $\mathrm{Zu} \mathrm{T}$ ist eine markante Veränderung sichtbar: die Fähigkeit, die zugrundeliegenden Faktoren in Bezug auf mentale Zustände zu reflektieren und selbständig zu erarbeiten, ist vorhanden, jedoch braucht Anna manchmal noch die Ermutigung dazu. Das Thema der Fremdunterbringung, sichtbar gemacht im Schweinchen Schwarzfuß-Test durch das Bild des „Karren“, der den Traum eines Schweinchens darstellt, in dem Schweine auf einen Karren geladen werden und dabei einen verzweifelten Gesichtsausdruck aufweisen, wird von Anna folgendermaßen bearbeitet: $\mathrm{Zu} \mathrm{T}$ integriert sie das Bild nicht in ihre Geschichte. Es wird an zweiter Stelle jener Bilder gelegt, die sie am wenigsten mag. Dabei erwähnt sie trocken, es würde ihr nicht gefallen, da das Schweinchen von 
seiner Familie getrennt wird. Eine Identifikation erfolgt explizit mit dem Schweinchen, das auf dem Karren weggebracht wird. $\mathrm{Zu} \mathrm{T}_{1}$ integriert sie das Bild in die Geschichte indem sie erzählt, dass das Schweinchen träumt, wie es von den Eltern getrennt wird. Eine Identifikation erfolgt nicht explizit. Interessant ist dennoch die Reihenfolge der Bilder in der Erzählung, denn der Traum erfolgt direkt nach der Schilderung eines Bildes, auf dem das Schweinchen von einem Gänserich verletzt wird. Zum letzten Testzeitpunkt $\left(\mathrm{T}_{2}\right)$ wird das Bild nicht in die Geschichte integriert, es wird unter die Bilder gereiht, die Anna nicht mag, jedoch weniger prominent und sehr nüchtern erzählt sie von dem Traum des Schweinchens, das „wegkommt“. Dabei identifiziert sie sich mit dem träumenden Schweinchen, das laut Anna 4 Jahre alt ist.

\section{David}

David ist zum ersten Testzeitpunkt neun Jahre alt und lebt seit seinem siebenten Lebensjahr in einer WG. $\mathrm{Zu}$ seiner älteren Schwester hat er keinen Kontakt. Seine Eltern sind Opioid- und Benzodiazepin abhängig. Die Abnahme erfolgte aufgrund gewalttätiger Auseinandersetzungen zwischen den Eltern und einer damit verbundenen Kindeswohlgefährdung. Der Kontakt zu den leiblichen Eltern blieb kontinuierlich seit der Fremdunterbringung bestehen. Seit 2018 wird über eine langsame Rückführung in die Familie nachgedacht. David verbringt Wochenenden und Schulferien bei seinen Eltern. Seit seiner Ankunft in der WG gab es keinen Bezugsbetreuungswechsel. Er wird seit drei Jahren von Katja betreut. Katja ist zu $\mathrm{T}_{0} 31$ Jahre alt. Bei dem Fragebogen der EBDD erzielt sie einen unterdurchschnittlichen Wert, der für eine restriktive Einstellung zum Drogenkonsum steht. Die Mentalisierungsfähigkeit ist zwar vorhanden, es zeigen sich Hinweise auf die Berücksichtigung mentaler Zustände, sie werden jedoch selten explizit gemacht. Ergänzend dazu zeigt auch das Ergebnis der Sekundärskala „Reflexibilität“ des ISK, dass sie sich mit ihrem Verhalten und dem Erleben des Verhaltens der Anderen in einem mittleren Maße auseinandersetzt.

Zum ersten Testzeitpunkt ist David aufgeweckt und interessiert, teilweise leicht unkonzentriert. Die Ergebnisse im CPM zeigen zu allen drei Testzeitpunkten eine deutlich unter dem Durchschnitt liegende intellektuelle Leistung. $\mathrm{Zu} \mathrm{T}_{2}$ finden sich in beiden Problemskalen des CBCL, vor allem in der Subskala „aggressives Verhalten“, Ergebnisse im Grenzbereich zum auffälligen Verhalten. In der kognitiven und affektiven, reflexiven Auseinandersetzung mit seiner Familie zeigt David $\mathrm{zu} \mathrm{T}_{0}$ keine Hinweise auf ein Vorhandensein einer reflexiven Funktion. Verhaltensbeschreibungen dominieren das Interview (CAI). $\mathrm{Zu}$ $\mathrm{T}_{1}$ zeigt sich eine deutliche Veränderung. Wie bei Anna, ist die Fähigkeit, die zugrundeliegenden Faktoren in Bezug auf mentale Zustände zu reflektieren und selbständig zu erarbeiten, vorhanden, wobei es manchmal noch die Ermutigung von außen dazu braucht. Zum letzten Testzeitpunkt ist David von der Wiederholung des Interviews genervt. Er beantwortet die Fragen knapper als $\mathrm{zu} \mathrm{T}_{1}$, die meisten Antworten beinhalten mehr spontane Berichte über den psychischen Zustand als elaborierte Zuschreibungen zu psychischem Erleben. Das Thema der Fremdunterbringung wird von David folgendermaßen bearbeitet: $\mathrm{Zu} \mathrm{T}_{0}$ integriert er das Bild des Karrens in seine Geschichte und verbalisiert die aggressive Komponente: „Ein Mensch schmeißt ein Schwein in die Kutsche“. Das Bild mag er von allen Bildern am wenigsten. Eine Identifikation mit dem Schwein auf dem Karren erfolgt nicht explizit. $\mathrm{Zu} \mathrm{T}_{1}$ integriert er das Bild nicht in die Geschichte. Er legt es wieder zu den Bildern, die er nicht mag und reiht direkt danach ein Bild, welches er mit den Worten „es hat Angst“ kommentiert. Zum letzten Testzeitpunkt $\left(\mathrm{T}_{2}\right)$ wird das Bild wieder in die Geschichte integriert, indem er den Traum als „etwas schlimmes“ beschreibt. Dazu identifiziert er sich mit dem träumenden Schweinchen mit der Begründung, dass er lieber davon träumt, weil es dann „nicht in echt passiert".

\section{Diskussion}

Besonders bei fremduntergebrachten Kindern muss der intergenerationale Kontext der Affektregulation in der Eltern-Kind-Beziehung, und in weiterer Folge in der Betreuer*in-Kind-Beziehung, berücksichtigt werden. Bisherige Forschungsergebnisse haben ein hohes $\mathrm{Ma}$ an intergenerationeller Stabilität bei Bindungsund Mentalisierungsfähigkeiten gezeigt (Fonagy et al. 2002). Vor allem Anna, die bereits im dritten Lebensjahr fremduntergebracht wurde, ist in der Entwicklung ihrer eigenen Mentalisierungsfähigkeit von der ihrer aktuellen Bezugspersonen abhängig. Die Mentalisierungsfähigkeit ihrer aktuellen Bezugsbetreuerin ist zwar, wie die Ergebnisse zeigen, vorhanden, über die Fähigkeiten der vorherigen Betreuer*innen ist nichts bekannt. Elternschaft gilt als wichtiger Modulator von Risiken, die auch der intergenerationalen Übertragung von Aggressionsmustern zugrunde liegen (Fonagy 2003). Beide Kinder waren Zeugen von häuslicher Gewalt und wurden vernachlässigt. Annas selbstkritische Haltung $\mathrm{zu} \mathrm{T}_{0}$ und $\mathrm{T}_{1}$ und ihre Reflexion darauf $\mathrm{zu} \mathrm{T}_{2}$ ist vergleichbar mit dem Konzept des „Alien-Selbst“ von Fonagy et al. (2002). Quälende Gefühle von Schlechtigkeit oder Wertlosigkeit, die als Teil des Selbst empfunden werden, aber nicht integriert sind, daher als „Fremd-Selbst“-Teile empfunden werden, können das Selbstwertgefühl dominieren. Versuche, sich an diese quälenden inneren Erfahrungen anzupassen, können sich auch in selbstschädigenden Verhaltensweisen manifestieren, die dazu dienen, Spannung abzubauen. Die Fähigkeit zu $\mathrm{T}_{2}$ zwischen innerer und äußerer Realität zu differenzieren und deutlich weniger Selbstkritik zu äußern, mag ein Aus- 
druck der Verbesserung der Mentalisierungsfähigkeit sein.

Basierend auf den Beobachtungsuntersuchungen des Trennungs- und Individuationsvorganges, welche Margaret Mahler 1959 in New York begann, beschrieb sie die gegenseitige Reflexion von Mutter und Kind als Grundlage der Identitätsentwicklung (vgl. Mahler 1963). Die Reaktion der Mutter auf die vom Säugling kommenden Signale ist in diesem Vorgang besonders wichtig. Sie postuliert, dass das Kleinkind, bei dem Loslösung und Individuation reibungslos verlaufen, sich im Falle einer kurzfristigen Trennung, mit seinen sich rasch entwickelnden Ich-Funktionen trösten kann. Es konzentriert sich dabei darauf, sich in Selbständigkeit zu üben. Sind die Phasen der Separation, Individuation und Wiederannäherung, wie bei Anna und David, jedoch durch eine fehlende Objektkonstanz gekennzeichnet, so entwickelt das Kind eine pathologische Angst vor dem Objektverlust, da die weniger gut ausgeprägten Ich-Funktionen nicht ausreichenden Schutz bieten können. Dies wird bei David in der Reflexion des Themas der Fremdunterbringung einerseits, wie auch in seiner Reaktion auf eine Schrittweise Rückführung in seine Familie deutlich. Davids Ergebnisse im unteren Grenzbereich der Subskala „aggressives Verhalten“ der CBCL könnten mit dem Ausbau der Besuchskontakte und seinen damit verbundenen ambivalenten Gefühlen zusammenhängen. Während der Teilnahme an der Gruppenintervention konnte anhand seines Verhaltens mehrmals die Sorge und die Freude bezüglich einer Intensivierung des Kontakts zu den Eltern beobachtet werden.

Zusammenfassend kann gesagt werden, dass es beiden Kindern 12 Monate nach der Teilnahme an der Gruppenintervention möglich ist, zugrundeliegende Faktoren in Bezug auf mentale Zustände zu reflektieren und selbständig zu erarbeiten, sowie auftauchende Affekte zu verbalisieren, anstatt von ihnen überwältigt, sie regressiv auszuagieren. Es kann davon ausgegangen werden, dass die Teilnahme an der Gruppenintervention einen positiven Beitrag $\mathrm{zu}$ dieser Entwicklung geleistet hat, auch wenn freilich eine Aussage über die Wirksamkeit dieser Intervention an dieser Stelle nicht getätigt werden kann. Die Weiterführung des Angebots und der Pilotstudie soll es ermöglichen, in naher Zukunft weitere Ergebnisse zu veröffentlichen.

Danksagung Die vorliegende Studie wird im Rahmen eines Praxisprojekts durchgeführt, für welches der Verein Dialog dankenswerterweise Projektmittel von den Gemeinsamen Gesundheitszielen aus dem Rahmen-Pharmavertrag (PHAR$M I G$ ) erhalten hat. Die PHARMIG hatte keinen Einfluss auf das Studiendesign, die Analysen oder die Interpretation der Ergebnisse.

Interessenkonflikt N. Springer und B. Lueger-Schuster geben an, dass kein Interessenkonflikt besteht.
Open Access Dieser Artikel wird unter der Creative Commons Namensnennung 4.0 International Lizenz veröffentlicht, welche die Nutzung, Vervielfältigung, Bearbeitung, Verbreitung und Wiedergabe in jeglichem Medium und Format erlaubt, sofern Sie den/die ursprünglichen Autor(en) und die Quelle ordnungsgemäß nennen, einen Link zur Creative Commons Lizenz beifügen und angeben, ob Änderungen vorgenommen wurden.

Die in diesem Artikel enthaltenen Bilder und sonstiges Drittmaterial unterliegen ebenfalls der genannten Creative Commons Lizenz, sofern sich aus der Abbildungslegende nichts anderes ergibt. Sofern das betreffende Material nicht unter der genannten Creative Commons Lizenz steht und die betreffende Handlung nicht nach gesetzlichen Vorschriften erlaubt ist, ist für die oben aufgeführten Weiterverwendungen des Materials die Einwilligung des jeweiligen Rechteinhabers einzuholen.

Weitere Details zur Lizenz entnehmen Sie bitte der Lizenzinformation auf http://creativecommons.org/licenses/by/4 . $0 /$ deed.de.

\section{Literatur}

Allen, J., Fonagy, P., \& Bateman, A. (2008). Mentalizing in clinical practice. Washington, DC: American Psychiatric Press.

Bateman, A., \& Fonagy, P. (2008). 8-year follow-up of patients treated for borderline personality disorder: mentalization-based treatment versus treatment as usual. The American Journal of Psychiatry, 165(5), 631-638. https://doi. org/10.1176/appi.ajp.2007.07040636.

Bekermans-Kranenburg, M. J., van Ijzendoorn, M.H., \& Juffer, F. (2003). Less is more: meta-analyses of sensitivity and attachment interventions in early childhood. Psychological Bulletin, 129, 195-215. https:// doi.org/10.1037/00332909.129.2.195.

Bolm, T. (2010). Mentalisieren und Mentalisierungsbasierte Therapie (MBT). Psychotherapie, 15(1), 58-62.

Bowlby,J. (1998). Attachment and loss: sadness and depression. Bd.3. London: Pimlico.

Cicchetti,D., Ragosch, F. A., \&Toth, S. L. (2006). Fosteringsecure attachment in infants in maltreating families through preventive interventions. Development and Psychopathology, 18, 623-649.

Dejko, et al. (2016). Characteristics of mentalization patterns in parents of children with difficulties in realizing developmental objectives of the latency stage - qualitative analysis results. Psychiatria Polska, 50(3), 597-606. https://doi. org/10.12740/PP/OnlineFirst/38904.

Ensink, K., Bégin, M., Normandin, L., Godbout, N., \& Fonagy, P. (2017). Mentalization and dissociation in the context of trauma: Implications for child psychopathology. Journal of Trauma and Dissociation, 18(1), 11-30. https:// doi. org/10.1080/15299732.2016.1172536.

European Monitoring Centre for Drugs and Drug Addiction (EMCDDA) (2020). Attitudes to drug use. Prevention evaluation instrument. http:/ / www.emcdda.europa. eu/html.cfm/index3426EN.html/. Zugegriffen: 3. Jänner 2020.

Fonagy, P. (1995). Playing with reality: the development of psychic reality and its malfunction in borderline personalities. International Journal of Psychoanalysis, 76, 39-44.

Fonagy, P. (2003). The developmental roots of violence in the failure of mentalization. In F. Pfafflin \& G. Adshead (Hrsg.), A matter of security. The application of attachment theory 
to forensic psychiatry and psychotherapy (S. 13-56). London: Jessica Kingsley Publishers.

Fonagy, P., Gergely, G., Jurist, E., \& Target, M. (2002). Affect regulation, mentalization and the development of the self. NewYork: Other Press.

Fonagy, P., Steele, H., \& Steele, M. (1991). Maternal representations of attachment during pregnancy predict the organization of infant-mother attachment at one year of age. Child Development, 62, 891-905.

Fonagy, P., Target, M., Steele, H., \& Steele, M. (1998). Reflecti$v e$ functioning manual. Version 5.0 for application to the adult attachment interviews. London: University College London.

Freud, S. (1909). Der Familienroman der Neurotiker. In Gesammelte Werke (1994) (Bd.5, S. 221-226). Frankfurtam Main: Fischer.

Jemerin, J. (2004). Latency and the capacity to reflect on mental states. Psychoanalytic Study of the Child, 59, 211-239.

Mahler, M. S. (1963). Thoughts about development and individuation. The Psychoanal. Study of the Child, 18,307-332.

Midgley, N., Ensink, K., Lindquist, K., Malberg, N., \& Muller, N. (2017). Mentalization-based treatment for children. A time-limited approach. Washington, DC: American Psychological Association.
Muller, N., Gerits, L., \& Siecker, I. (2012). Mentalization-based therapies with adopted children and their families. In N. Midgley \& I. Vrouva (Hrsg.), Minding the child: Mentalization-based interventions with children, young people and their families (S. 113-130). London: Routledge.

Ostler, T., Bahar, O.S., \& Jessee, A. (2010). Mentalization in children exposed to parental methamphetamine abuse: relations to children's mental health and behavioural outcomes. Attachment \& Human Development, 12(3), 193-207.https://doi.org/10.1080/14616731003759666.

Suchman, N.E., DeCoste, C., Leigh, D., \& Borelli, J. (2010). Reflective functioning in mothers with drug use disorders: implicationsfordyadicinteractions withinfants and toddlers. Attachment \& Human Development, 12, 567-585. https://doi.org/10.1080/14616734.2010.501988.

Thomsen, T., Lessing, N., \& Greve, W. (2017). Transgenerationale Emotionsregulation. Kindheit und Entwicklung, 26, 7-18. https://doi.org/10.1026/0942-5403/a000211.

Winnicott, D. W. (1971). Playing and reality. London: Tavistock Publications.

Hinweis des Verlags Der Verlag bleibt in Hinblick auf geografische Zuordnungen und Gebietsbezeichnungen in veröffentlichten Karten und Institutsadressen neutral. 